\title{
NUMERICAL MODELING OF A DUAL PONTOON FLOATING STRUCTURE WITH A LIQUID CONTAINER
}

\author{
Chai-Cheng Huang \\ Department of Marine Environment and Engineering, Nation Sun Yat-sne University, Kaohsiung, Taiwan, R.O.C., \\ cchuang@mail.nsysu.edu.tw \\ Chih-Ting Feng \\ Department of Marine Environment and Engineering, Nation Sun Yat-sne University, Kaohsiung, Taiwan, R.O.C. \\ Hung-Jie Tang \\ Tainan Hydraulics Laboratory, National Cheng Kung University, Tainan, Taiwan, R.O.C.
}

Follow this and additional works at: https://jmstt.ntou.edu.tw/journal

Part of the Engineering Commons

\section{Recommended Citation}

Huang, Chai-Cheng; Feng, Chih-Ting; and Tang, Hung-Jie (2011) "NUMERICAL MODELING OF A DUAL PONTOON FLOATING STRUCTURE WITH A LIQUID CONTAINER," Journal of Marine Science and Technology. Vol. 19: Iss. 6, Article 1.

DOI: $10.51400 / 2709-6998.2199$

Available at: https://jmstt.ntou.edu.tw/journal/vol19/iss6/1

This Research Article is brought to you for free and open access by Journal of Marine Science and Technology. It has been accepted for inclusion in Journal of Marine Science and Technology by an authorized editor of Journal of Marine Science and Technology. 


\section{NUMERICAL MODELING OF A DUAL PONTOON FLOATING STRUCTURE WITH A}

LIQUID CONTAINER

\section{Acknowledgements}

The authors acknowledge the support in part by AsiaPacific Ocean Research Center, NSYSU, and the National Science Council (NSC98-2221-E-110-087), Taiwan. 


\title{
NUMERICAL MODELING OF A DUAL PONTOON FLOATING STRUCTURE WITH A LIQUID CONTAINER
}

\author{
Chai-Cheng Huang*, Chih-Ting Feng*, and Hung-Jie Tang**
}

Key words: DPFS, BIEM, NWT, sloshing, acceleration potential method, modal decomposition method.

\begin{abstract}
A numerical model is developed to investigate the waveinduced dynamic properties of a dual pontoon floating structure (DPFS) with a liquid container on the top. This model has to deal with two kinds of fluid domain: one is the incident wave domain, while the other is the excited fluid domain in a sloshing container. The boundary integral equation method (BIEM) with linear element scheme is applied to forming a 2D fully nonlinear numerical wave tank (NWT). The secondorder Stokes wave theory is adopted to give the velocity on the input boundary. The velocity potential and acceleration potential are solved simultaneously to obtain the contact forces on the DPFS as well as the liquid container. Summing the total external forces on the floating platform, we may form a system of motion equations. To establish an accurate and yet efficient numerical model is the goal of this paper, and it may lay a good foundation for the further study on the renewable wave energy devices or structural motion damping apparatus.
\end{abstract}

\section{INTRODUCTION}

Offshore platforms are important structures for developing or securing the natural resources from the ocean. Generally, they could be classified into two categories: One is supported by columns mounted on the sea bed, and the other is moored floating structures. The former has been extensively studied by many researchers such as MacCamy and Fuchs [13], Chakrabarti [1], Perrey-Debain et al. [15], and more recently by Chen et al. [2] who developed a very efficient and accurate technique to calculate the interactions between water waves

Paper submitted 11/06/09; revised 04/26/10; accepted 06/22/10. Author for correspondence: Chai-Cheng Huang (e-mail: cchuang@mail.nsysu.edu.tw).

*Department of Marine Environment and Engineering, Nation Sun Yat-sne University, Kaohsiung, Taiwan, R.O.C.

**Tainan Hydraulics Laboratory, National Cheng Kung University, Tainan, Taiwan, R.O.C. and pile groups. On the other hand, floating platforms have been investigated by numerous researchers. For example, Nojiri and Murayama [14] performed an experimental study, while Tanizawa and Minami [19], Koo and Kim [10], and Huang et al. [16] developed numerical models based on a boundary integral equation method (BIEM) to deal with the problems. In recent years, sloshing is often used in floating structures as damping mechanism, such as tuned liquid column damper (TLCD), which can be found in Gao et al. [4], Hitchcock et al. [6,7], and Lee et al. [11] etc. In addition, Williams and Abul-Azm [22] investigated numerically the wave reflection properties of a dual pontoon floating structure. Weng and Chou [21] used both BIEM-based numerical model and physical model to investigate the dynamic responses of body motion and wave reflection. However, the flow field in the above models was limited to the scope of a linear wave theory.

The nonlinear wave-body interaction problem of a floating single pontoon structure was studied by Isaacson [9] using BIEM. Lately, the numerical simulation of nonlinear wavebody interaction was investigated by a fully nonlinear numerical wave tank (Tanizawa [17]; Koo and Kim [10]; Huang et al. [16] etc.). In this paper, the boundary integral equation (BIE) is formulated by using the linear element technique. Subsequently, the free surface variation is traced by the Mixed Eulerian and Lagrangian method (MEL), Longuet-Higgins and Cokelet [12], and the 4th order Runge-Kutta method (RK4), meanwhile a node-regridding technique is applied by using a cubic spline scheme to preventing free surface nodes moving too close to each other. Two numerical damping zones (Cointe [3] and Tanizawa [18]) are implemented on both ends of numerical wave tank to absorb or dissipate the reflected and transmitted waves due to wave-body interaction. The instantaneous hydrodynamic force is calculated by an acceleration potential method (Tanizawa [17]) and a modal decomposition method (Vinje and Brevig [20]).

The objective of this paper is to establish a numerical model to study the wave-induced dynamic properties of a dual pontoon floating structure with a liquid container on the top, and it may set a foundation for the further study on the renewable wave energy devices or structural motion damping apparatus. 


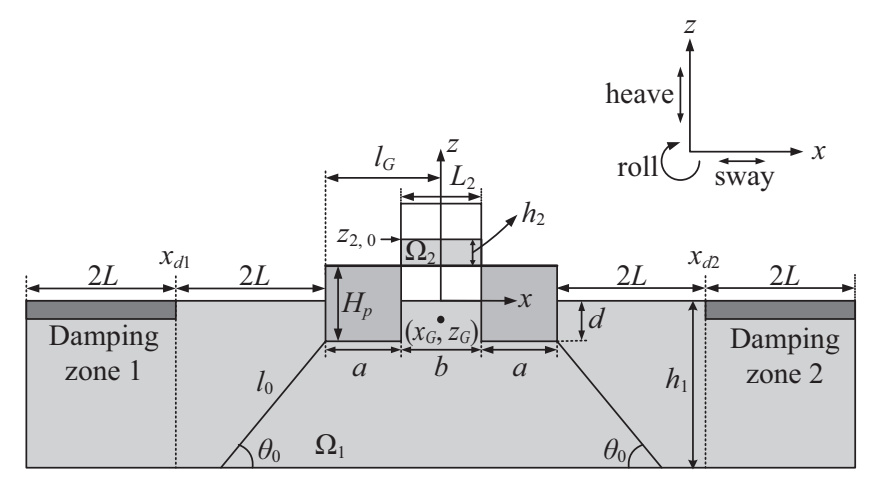

Fig. 1. The definition of floating structure sketch and its degree of freedoms.

\section{NUMERICAL MODEL}

A dual pontoon floating structure consisting of a pair of floating cylinders and a liquid container on the top is restrained by a linear symmetric mooring system, as show in Fig. 1, where $\Omega_{1}$ is the fluid domain of numerical wave tank, $\Omega_{2}$ is the fluid domain of the liquid container, $a$ is the width of the pontoon, $b$ is the spacing between two pontoons, $d$ is the draft, $\left(x_{G}, z_{G}\right)$ is the position of gravity center, $H_{p}$ is the height of the pontoon, $l_{G}$ is the roll moment arm, $\theta_{0}$ is the mooring angle, $l_{0}$ is the original length of mooring line, $L_{2}$ is the width of the liquid container, $h_{2}$ is the water depth of the liquid container, and $z_{2,0}$ is the elevation of free surface in $\Omega_{2}$ at $t=0$. This floating structure is deployed in a numerical wave tank where the water depth $h_{1}$ is held constant. Two numerical damping zones are deployed at both ends of the wave tank to dissipate reflected and transmitted waves, where $x_{d 1}$ and $x_{d 2}$ are the entrance positions. Since the target model is a $2 \mathrm{D}$ model, only three degree of freedoms are involved in the calculation, shown in Fig. 1, where the horizontal motion is sway, the vertical motion is heave, and the rotational motion is roll.

\section{Governing Equations}

The flow field is assumed to be incompressible, inviscid and irrotational. Thus, the velocity potential satisfies Laplace equation,

$$
\nabla^{2} \phi_{k}=0 \quad \text { at } \Omega_{k}(t)
$$

where the subscript, $k=1,2$, indicates the fluid domain $\Omega_{1}$ and $\Omega_{2}$ respectively.

Incorporated Eq. (1) into Green second identity, the velocity potential in the fluid domain can be determined by solving the following BIE:

$$
\alpha_{k, i} \phi_{k, i}=\int_{\Omega_{k}}\left(\frac{\partial G_{k, i j}}{\partial n} \phi_{k, j}-G_{k, i j} \frac{\partial \phi_{k, j}}{\partial n}\right) d \Gamma_{k} \quad \text { at } \Omega_{k}(t)
$$

$G_{i j}=\ln r_{i j} / 2 \pi$ is the fundamental solution satisfying Laplace equation and representing a flow field generated by a concentrated unit source acting at $i$ th source point, $r_{i j}$ is the distance from source point $\left(x_{i}, z_{i}\right)$ to field point $\left(x_{j}, z_{j}\right)$ and $\alpha_{i}$ is the normalized internal solid angle between two boundary elements. In this model, the linear element scheme and 6-point Gaussian quadrature integration method are adopted to solve the BIE.

\section{Inflow Boundary Condition}

Based on the continuity of velocity, theoretical particle velocity profile can be used to specify the boundary value along the inflow boundary. For nonlinear regular waves, the second-order Stokes wave is adopted to prevent the mismatch between input velocity profiles and real water particle velocity, as described in Koo and Kim [10].

$$
\begin{aligned}
\frac{\partial \phi_{1}}{\partial n} & =-\frac{\partial \phi_{I}}{\partial x} \\
& =-\left[\begin{array}{l}
\frac{g A k}{\sigma} \frac{\cosh k\left(z+h_{1}\right)}{\cosh k h_{1}} \cos (k x-\sigma t) \\
+\frac{3}{4} A^{2} k \sigma \frac{\cosh 2 k\left(z+h_{1}\right)}{\sinh ^{4} k h_{1}} \cos 2(k x-\sigma t)
\end{array}\right] f_{m} \quad \text { on } \Gamma_{1, I}
\end{aligned}
$$

where $A, k, \sigma$ are the amplitude, wave number, and angular frequency, respectively. $g$ is the gravitational acceleration, $t$ is time. For numerical stability, the modulation function $f_{m}$ is used to prevent impulse-like behavior of wavemaker, and it is written as

$$
f_{m}(t)= \begin{cases}\frac{1}{2}\left[1-\cos \left(\frac{\pi t}{T_{m}}\right)\right] & \text { for } t<T_{m} \\ 1 & \text { for } t \geq T_{m}\end{cases}
$$

\section{Free Surface Boundary Condition}

One of the most popular and successful approaches in the fully nonlinear free surface simulation is the MEL method, which was firstly presented by Longuet-Higgins and Cokelet [12]. In this method, the kinematic and dynamic free surface boundary conditions are transformed into the Lagrangian frame. To obtain proper numerical solutions for wave propagation in a finite computational fluid domain, the scheme so-called numerical damping zones deployed at both ends of wave tank are used to absorb the transmitted wave energy at the end of wave tank as well as to dissipate the reflected waves in front of the input boundary. Following Cointe [3] and Tanizawa and Minami [19], the numerical damping zones are incorporated into the free surface boundary conditions as, 


$$
\begin{aligned}
& \begin{cases}\frac{d x}{d t}=\frac{\partial \phi_{1}}{\partial x} & \text { on } \Gamma_{1, f} \\
\frac{d z}{d t}=\frac{\partial \phi_{1}}{\partial z}-v(x)\left(z-z_{e}\right) & \frac{d \phi}{d t}=-g z+\frac{1}{2}\left|\nabla \phi_{1}\right|^{2}-v(x)\left(\phi_{1}-\phi_{e}\right)\end{cases} \\
& \begin{cases}\frac{d x}{d t}=\frac{\partial \phi_{2}}{\partial x} & \text { on } \Gamma_{2, f} \\
\frac{d z}{d t}=\frac{\partial \phi_{2}}{\partial z} & \frac{1}{d \phi_{2}} \\
\frac{d t}{d t}=-g\left(z-z_{2,0}\right)+\frac{1}{2}\left|\nabla \phi_{2}\right|^{2} & \end{cases}
\end{aligned}
$$

where $v(x)$ is the damping coefficient of numerical damping zone given by

$$
v(x)= \begin{cases}\alpha_{d} \sigma\left[\left(x_{d 1}-x\right) / L\right]^{2} & x \leq x_{d 1} \\ \alpha_{d} \sigma\left[\left(x-x_{d 2}\right) / L\right]^{2} & x \geq x_{d 2} \\ 0 & \text { otherwise }\end{cases}
$$

where $\alpha_{d}$ is the dimensionless parameter for the strength of the damping zone, and it is set to 1 in the present model. $L$ is wavelength of the input wave. $x_{d 1}$ and $x_{d 2}$ are the entrance position of each damping zones shown in Fig. 1. $z_{e}$ and $\phi_{e}$ in Eq. (5) are the entrance wave elevation and the potential in the front damping zone and thus they only exist in the region $x \leq$ $x_{d 1}$. Tanizawa and Minami [19] applied a damping zone which works as an absorber in front of input boundary to dissipate the wave energy re-reflected by the structure. He pointed out that this solution can be computed by numerical simulation of the waves without structures in the tank. For practical purpose, the nonlinear analytical solution is a good substitution and therefore, in this model, the entrance potential and wave elevation are obtained from the second-order Stokes wave theory as

$$
\left\{\begin{aligned}
\phi_{e}= & \frac{A g}{\sigma} \frac{\cosh k(z+h)}{\cosh k h} \sin (k x-\sigma t) \\
& +\frac{3}{8} A^{2} \sigma \frac{\cosh 2 k(z+h)}{\sinh ^{4} k h} \sin 2(k x-\sigma t) \\
z_{e}= & A \cos (k x-\sigma t)+\frac{k A^{2} \cosh k h}{4 \sinh ^{3} k h}(2+\cosh 2 k h) \cos 2(k x-\sigma t)
\end{aligned}\right.
$$

Additionally, the nodal velocities in the right hand side of Eq. (5) are obtained by using the cubic spline scheme in the curvilinear coordinate system and coordinate transformation scheme, $s n$-coordinates transformed to $x y$-coordinates, referring to Tang and Huang et al. [16]; while the corner problem between free surface and body surface is treated according to Grilli and Svendsen [5].
Once the horizontal and vertical velocities are known, the total derivative equations of Eq. (5) can be used to predict the nodal new position and its corresponding potential on the free surface boundary, by employing the RK4 method as time marching scheme. Repeat this process until the simulation reaches the steady-state condition.

\section{Body Surface Boundary Condition}

In this model, the body surface $\left(\Gamma_{1, s}\right.$ and $\left.\Gamma_{2, s}\right)$ is considered as impermeable. Therefore, the fluid normal velocity on the body is equal to the normal velocity of the body surface

$$
\frac{\partial \phi_{k}}{\partial n}=n_{1} \dot{x}_{G}+n_{2} \dot{z}_{G}+n_{3} \dot{\theta}_{G} \quad \text { on } \Gamma_{k, s}
$$

where $\left(n_{1}, n_{2}, n_{3}\right)=\left(n_{x}, n_{z}, r_{z} n_{x}-r_{x} n_{z}\right)$ are the unit normal vector on the body boundary; $\left(r_{x}, r_{z}\right)=\left(x-x_{G}, z-z_{G}\right)$ are the position vector from gravity center to body surface; subscript $G$ is the gravity center of the body; $\left(\dot{x}_{G}, \dot{z}_{G}\right)$ are the translational velocity of the gravity center in $x$ and $z$ axes (sway and heave motion); $\dot{\theta}_{G}$ is the angular velocity in the $y$ axis (roll motion).

\section{Rigid Boundary Condition}

At the end-wall $\left(\Gamma_{1, w}\right)$ and bottom $\left(\Gamma_{1, b}\right)$ of wave tank, boundary conditions are considered as impermeable. Therefore, the normal velocities are set to zeros.

$$
\frac{\partial \phi_{1}}{\partial n}=0 \text { on } \Gamma_{1, b} \text { and } \Gamma_{1, w}
$$

\section{Wave Forces on the Body}

The hydrodynamic forces on the body can be calculated by integrated the pressure around the wetted body surface as

$$
\left\{\begin{array}{l}
\boldsymbol{F}_{f}=\int_{\Gamma_{1, s}} P_{1} \boldsymbol{n} d s+\int_{\Gamma_{2, s}} P_{2} \boldsymbol{n} d s \\
\boldsymbol{M}_{f}=\int_{\Gamma_{1, s}} P_{1} \boldsymbol{r} \times \boldsymbol{n} d s+\int_{\Gamma_{2, s}} P_{2} \boldsymbol{r} \times \boldsymbol{n} d s
\end{array}\right.
$$

where $\boldsymbol{F}$ and $\boldsymbol{M}$ are the hydrodynamic force and moment, respectively. $\rho$ is the water density, $\boldsymbol{n}$ is the unit normal vector on the body surface and $\boldsymbol{r}$ is the position vector from gravity center to body surface.

The pressure from the fluid domain $\Omega_{1}$ and $\Omega_{2}$ are described as

$$
\left\{\begin{array}{l}
P_{1}=-\rho_{1}\left(\phi_{1, t}+g z+\frac{1}{2}\left|\nabla \phi_{1}\right|^{2}\right) \\
P_{2}=-\rho_{2}\left(\phi_{2, t}+g\left(z-z_{2,0}\right)+\frac{1}{2}\left|\nabla \phi_{2}\right|^{2}\right)
\end{array}\right.
$$




\section{Acceleration Potential Method}

To calculate instantaneous floating body motions, we have to solve the simultaneous equation of fluid and floating body motion. For this purpose, we used the acceleration potential method formulated by Tanizawa [17] to calculate the instantaneous $\phi_{t}$ of Eq. (12) by solving the BIE with the governing equation of acceleration field,

$$
\nabla^{2} \phi_{k, t}=0
$$

The body-surface boundary condition in acceleration field is described as

$$
\frac{\partial \phi_{k, t}}{\partial n}=n_{1} \ddot{x}_{G}+n_{2} \ddot{z}_{G}+n_{3} \ddot{\theta}_{G}+q_{k} \quad \text { on } \Gamma_{k, s}
$$

where $\left(\ddot{x}_{G}, \ddot{z}_{G}\right)$ are the translational acceleration of the gravity center in $x$ and $z$ axes; $\ddot{\theta}_{G}$ is the angular acceleration in $y$ axis. And $q$ is described as

$$
\begin{aligned}
q_{k}= & n_{1} \dot{\theta}_{G}\left(r_{x} \dot{\theta}_{G}-2 \dot{z}_{G}+2 \frac{\partial \phi_{k}}{\partial z}\right)+n_{2} \dot{\theta}_{G}\left(r_{z} \dot{\theta}_{G}+2 \dot{x}_{G}-2 \frac{\partial \phi_{k}}{\partial x}\right) \\
& +k_{n}\left[\left(\frac{\partial \phi_{k}}{\partial x}-\dot{x}_{G}-r_{z} \dot{\theta}_{G}\right)^{2}+\left(\frac{\partial \phi_{k}}{\partial z}-\dot{z}_{G}+r_{x} \dot{\theta}_{G}\right)^{2}\right] \\
& -k_{n}\left[\left(\frac{\partial \phi_{k}}{\partial x}\right)^{2}+\left(\frac{\partial \phi_{k}}{\partial z}\right)^{2}\right]-\frac{\partial \phi_{k}}{\partial s} \frac{\partial^{2} \phi_{k}}{\partial n \partial s}+\frac{\partial \phi_{k}}{\partial n} \frac{\partial^{2} \phi_{k}}{\partial s^{2}}
\end{aligned}
$$

where $k_{n}=1 / \rho *$ is normal curvature along the $s$ direction of the body surface; $\rho *$ is the radius of curvature.

\section{Modal Decomposition Method}

The idea of the modal decomposition method was first introduced by Vinje and Brevig [20]. This method is utilized to solve the BIE of acceleration field. The acceleration field is decomposed into four modes. The first three modes are associated with the unit acceleration in three degrees of freedom, i.e., sway, heave, and roll motions (radiation modes) and the fourth mode is due to the incoming wave scattered by the fixed structure (diffraction mode). Each mode can be obtained by solving the respective BIE. Using these four modes and the body-motion equation, body acceleration can be determined. The $\phi_{t}$ for 2D case is given by

$$
\phi_{k, t}=\sum_{m=1}^{3} a_{m} \varphi_{k, m}+\varphi_{k, 4}
$$

where $a_{m}$ is the $m$ th mode component of generalized body acceleration $(1=$ sway, 2 = heave, $3=$ roll $)$.

The boundary conditions in acceleration field for each mode is given as

$$
\begin{gathered}
\frac{\partial \varphi_{k, m}}{\partial n}=\left\{\begin{array}{cc}
n_{m} & m=1,2,3 \\
q_{k} & m=4
\end{array} \quad \text { on } \Gamma_{k, s}\right. \\
\varphi_{1, m}=\left\{\begin{array}{cc}
0 & m=1,2,3 \\
-g z-\frac{1}{2}\left|\nabla \phi_{1}\right|^{2} & m=4
\end{array} \quad \text { on } \Gamma_{1, f}\right. \\
\varphi_{2, m}=\left\{\begin{array}{c}
0 \quad m=1,2,3 \\
-g\left(z-z_{2,0}\right)-\frac{1}{2}\left|\nabla \phi_{2}\right|^{2} \quad m=4 \quad \text { on } \Gamma_{2, f}
\end{array}\right. \\
\frac{\partial \varphi_{1, m}}{\partial n}=0 \quad(m=1,2,3) \quad \text { on } \Gamma_{1, I} \\
\\
\frac{\partial n}{\partial n} \quad(m=1 \sim 4) \quad \text { on } \Gamma_{1, b} \text { and } \Gamma_{1, w}
\end{gathered}
$$

The inflow boundary condition for mode 4 is obtained from the 2nd-order Stokes wave theory,

$$
\frac{\partial \varphi_{1,4}}{\partial n}=-\left[\begin{array}{l}
g A k \frac{\cosh k\left(z+h_{1}\right)}{\cosh k h_{1}} \sin (k x-\sigma t) \\
+\frac{3}{2} A^{2} k \sigma^{2} \frac{\cosh 2 k\left(z+h_{1}\right)}{\sinh ^{4} k h_{1}} \sin 2(k x-\sigma t)
\end{array}\right] \text { on } \Gamma_{1, I}
$$

After solving the BIE for each mode, we would obtain the all variable values, $\varphi_{m}$ and $\partial \varphi_{m} / \partial n$ on the boundaries. Then the remaining unknown values on Eq. (16) are $a_{m}$ only.

Substituting Eq. (16) into Eq. (11), and then combining with Newton's second law including the wave hydrodynamic forces and the other extra forces (subscript $e$ ), e.g. gravitational force, damping force, and the mooring cable's restoring force, the equations of motion become

$$
\left\{\begin{aligned}
m a_{1}= & \int_{\Gamma_{1, s}}-\rho\left(a_{1} \varphi_{1,1}+a_{2} \varphi_{1,2}+a_{3} \varphi_{1,3}+\varphi_{1,4}+g z+\frac{1}{2}\left|\nabla \phi_{1}\right|^{2}\right) n_{1} d \Gamma \\
& +\int_{\Gamma_{2, s}}-\rho\left(a_{1} \varphi_{2,1}+a_{2} \varphi_{2,2}+a_{3} \varphi_{2,3}+\varphi_{2,4}+g\left(z-z_{2,0}\right)+\frac{1}{2}\left|\nabla \phi_{2}\right|^{2}\right) n_{1} d \Gamma \\
& +F_{e x} \\
m a_{2}= & \int_{\Gamma_{1, s}}-\rho\left(a_{1} \varphi_{1,1}+a_{2} \varphi_{1,2}+a_{3} \varphi_{1,3}+\varphi_{1,4}+g z+\frac{1}{2}\left|\nabla \phi_{1}\right|^{2}\right) n_{2} d \Gamma \\
& +\int_{\Gamma_{2, s}}-\rho\left(a_{1} \varphi_{2,1}+a_{2} \varphi_{2,2}+a_{3} \varphi_{2,3}+\varphi_{2,4}+g\left(z-z_{2,0}\right)+\frac{1}{2}\left|\nabla \phi_{2}\right|^{2}\right) n_{2} d \Gamma \\
& +F_{e z} \\
I_{G} a_{3}= & \int_{\Gamma_{1, s}}-\rho\left(a_{1} \varphi_{1,1}+a_{2} \varphi_{1,2}+a_{3} \varphi_{1,3}+\varphi_{1,4}+g z+\frac{1}{2}\left|\nabla \phi_{1}\right|^{2}\right) n_{3} d \Gamma \\
& +\int_{\Gamma_{2, s}}-\rho\left(a_{1} \varphi_{2,1}+a_{2} \varphi_{2,2}+a_{3} \varphi_{2,3}+\varphi_{2,4}+g\left(z-z_{2,0}\right)+\frac{1}{2}\left|\nabla \phi_{2}\right|^{2}\right) n_{3} d \Gamma \\
& +M_{e y}
\end{aligned}\right.
$$

After solving Eq. (22), the generalized acceleration $a_{m}$ can be obtained. And then $\phi_{t}$ is finally determined from Eq. (16). 
Table 1. Conditions for the numerical simulations.

\begin{tabular}{|c|c|c|c|c|}
\hline Parameter & Total mass & Draft & $\begin{array}{c}\text { Width of the } \\
\text { liquid } \\
\text { container }\end{array}$ & $\begin{array}{c}\text { Water depth } \\
\text { in the liquid } \\
\text { container }\end{array}$ \\
\hline case 1 & $m_{\text {Total }}=m_{1}$ & $d / h_{1}=0.3$ & \multicolumn{2}{|c|}{ without liquid container } \\
\hline case 2 & $m_{\text {Total }}=m_{1}+m_{2}$ & $d / h_{1}=0.3$ & $L_{2} / h_{1}=0.25$ & $h_{2} / h_{1}=0.05$ \\
\hline case 3 & $m_{\text {Total }}=m_{1}+m_{2}$ & $d / h_{1}=0.3$ & $L_{2} / h_{1}=0.5$ & $h_{2} / h_{1}=0.025$ \\
\hline case 4 & $m_{\text {Total }}=m_{1}+m_{2}$ & $d / h_{1}=0.4$ & $L_{2} / h_{1}=0.5$ & $h_{2} / h_{1}=0.025$ \\
\hline
\end{tabular}

\section{Mooring Force}

The mooring system is considered as linear and symmetric (see Fig. 1) with the spring constant $K$, and the wave hydrodynamic forces on the mooring line is neglected. The pretension force of this mooring system is written as

$$
F_{T_{0}}=\frac{2 \rho \operatorname{gad} \lambda-m g}{2 \sin \theta_{0}}
$$

where $\lambda$ is the length of the floating dual pontoon structure in $y$-direction, and in this model $\lambda=1 \mathrm{~m}$.

\section{RESULTS AND DISCUSSIONS}

The normalized input parameters of this simulation are as follows: $h_{1}=1.0 \mathrm{~m}, m_{1}=2 v_{1} \rho a d, m_{2}=2 \rho L_{2} h_{2}, b / h_{1}=0.5$, $a / h_{1}=0.25, H_{p} / h_{1}=0.45, z_{G}=-0.5 d, I_{G}=\frac{1}{2} v_{2} \rho a d\left[\frac{1}{3} d^{2}+\right.$ $\left.\frac{1}{3} a^{2}+(a+b)^{2}\right], \theta_{0}=60^{\circ}, l_{G}=a+b / 2, K / \rho g h=0.06, h_{2} / h_{1}=$ 0.05 . Coefficients $v_{1}$ and $v_{2}$ are dependent on the density and shape of the structure, and are simply given the same constant value 0.9 in this study. In order to explore how the draft and the size of liquid container affect the dynamic responses of floating structure, a series of conditions are described in Table 1 , and except case 1 , other cases set the total mass of the liquid in the container equal in each simulation, i.e. $L_{2} h_{2}=$ const .

\section{Comparisons of RAO}

In this simulation, the response amplitude operator (RAO) is adopted to describe the relation between the amplitude of body gravity center motions $\left(x_{G}, z_{G}, \theta_{G}\right)$ and incident wave amplitude $(A)$, as shown in Eq. (24).

$$
\left\{\begin{array}{l}
\text { Normalized sway motion, RAO }:\left(x_{G} / A\right) \\
\text { Normalized heave motion, } \mathrm{RAO}:\left(z_{G} / A\right) \\
\text { Normalized roll motion, } \mathrm{RAO}:\left(l_{G} \theta_{G} / A\right) \\
\text { Normalized tension force, } \mathrm{RAO}:\left(F_{T} / K A\right)
\end{array}\right.
$$

\section{Influence of Liquid Container}

Fig. 2 shows that the sway RAO decreases as the frequency increase for all three cases, although a sudden drop occurred

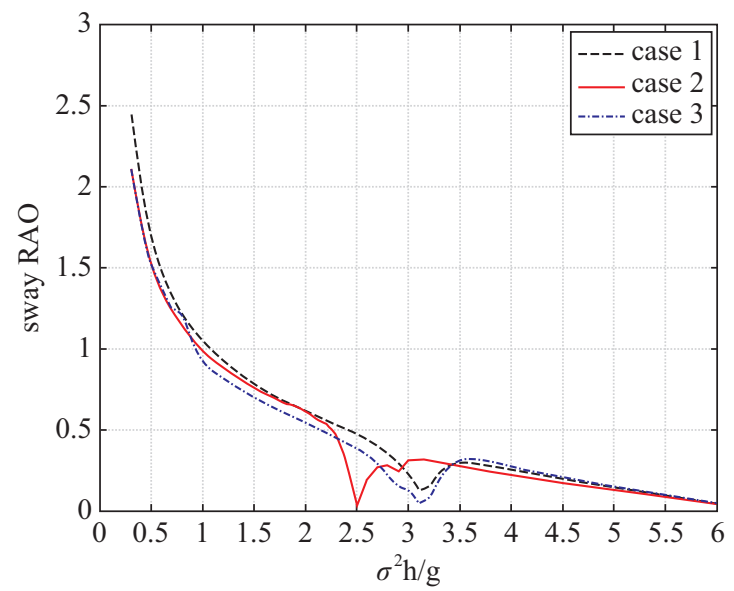

Fig. 2. Comparison of sway RAO with different liquid container conditions.

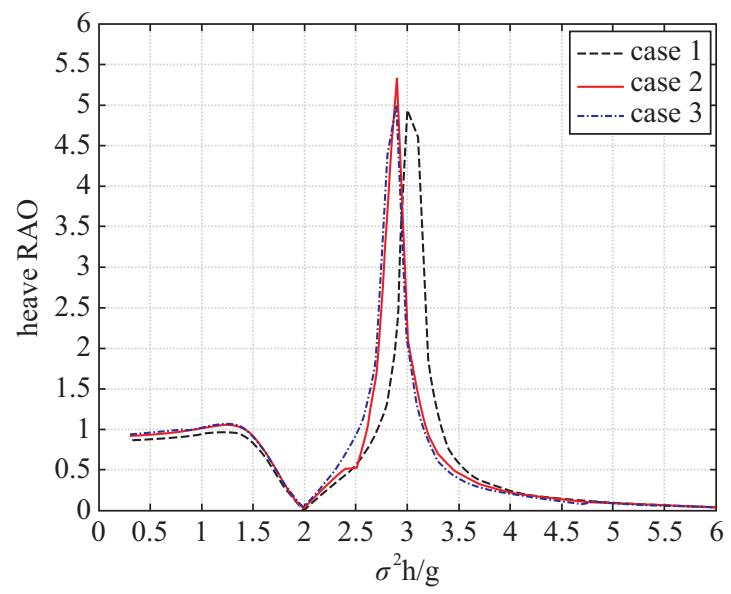

Fig. 3. Comparison of heave RAO with different liquid container conditions.

in the trends. It seems that the influence of liquid container on the sway motion in minor and slightly down shift in frequency, which may be attributed to the additional liquid mass added into the system. In heave RAO, Fig. 3 indicates that the response curves for three cases are similar in the shape. Though a downshift phenomenon in the resonant frequency for both cases with liquid container has discovered, the peak of resonant frequency in the case 2 , with deeper water depth in container, seems generating higher peak than the case 3 which has shallower water depth but wider width. While in roll RAO (see Fig. 4), the peak response of case 2 is significant downshift and creates higher peak value. Fig. 5 reveals the comparison of sea-side tension RAO. The result shows that both cases 2 and 3 have two resonant peaks in frequency domain, but the peaks are much lower than the case 1 without liquid container on the top of floating structure. This is most likely due to the sloshing effect against the structural motion, and thus reduces the mooring tension. 


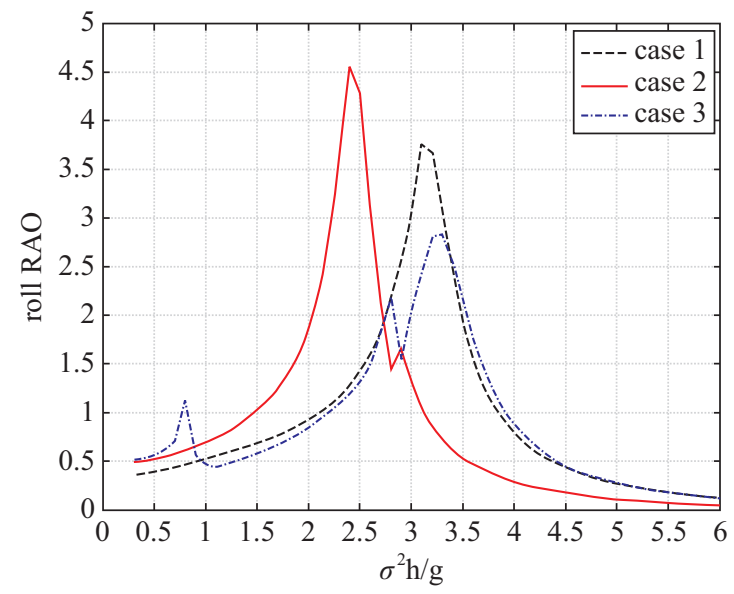

Fig. 4. Comparison of roll RAO with different liquid container conditions.

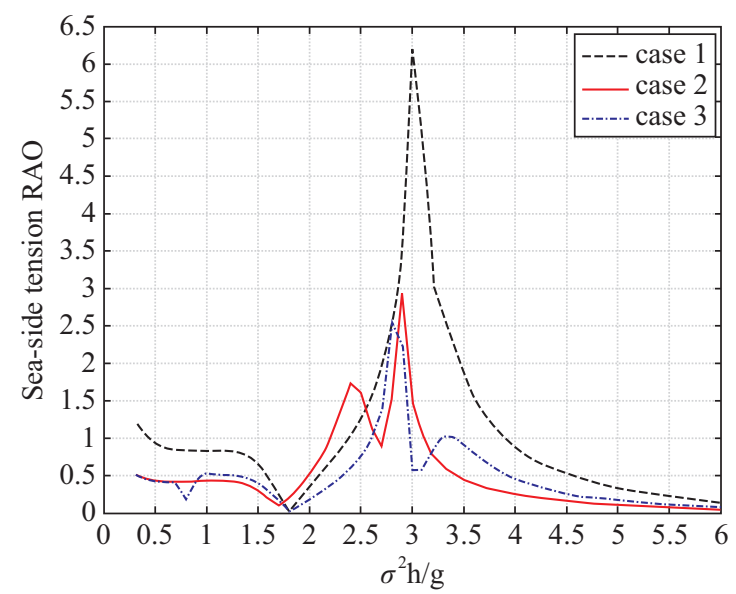

Fig. 5. Comparison of sea-side tension RAO with different liquid container conditions.

\section{Influence of the Depth of Draft}

In this section, we focus on the influence of the depth of draft on structural dynamic responses. Figs. 6-8 show that the floating structure with deeper draft the resonant frequencies in each mode of motion shift to lower frequency region and with higher peaks; the phenomenon is caused by that the total mass of the dual pontoon system increases with draft according the formula described at the beginning of RESULTS and DISCUSSIONS. Fig. 9 reveals the comparison of sea-side tension RAO. The result shows that the resonant frequencies also shift to low frequency region for the case with deeper draft, and also generating much higher peak than its counterpart with shallower draft.

\section{CONCLUSIONS}

In this study, a fully nonlinear numerical wave tank is developed to investigate the dynamic responses of a moored floating dual pontoon structure in waves. The mooring system

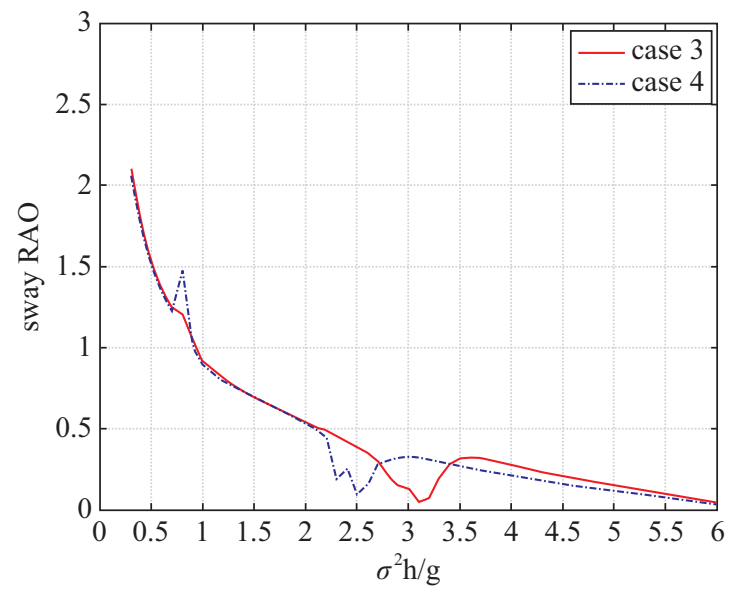

Fig. 6. Comparison of sway RAO with different drafts.

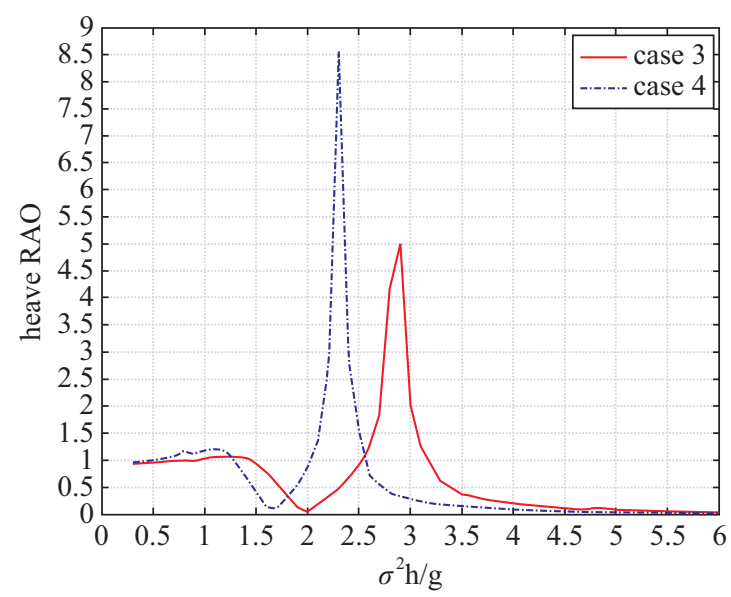

Fig. 7. Comparison of heave RAO with different drafts.

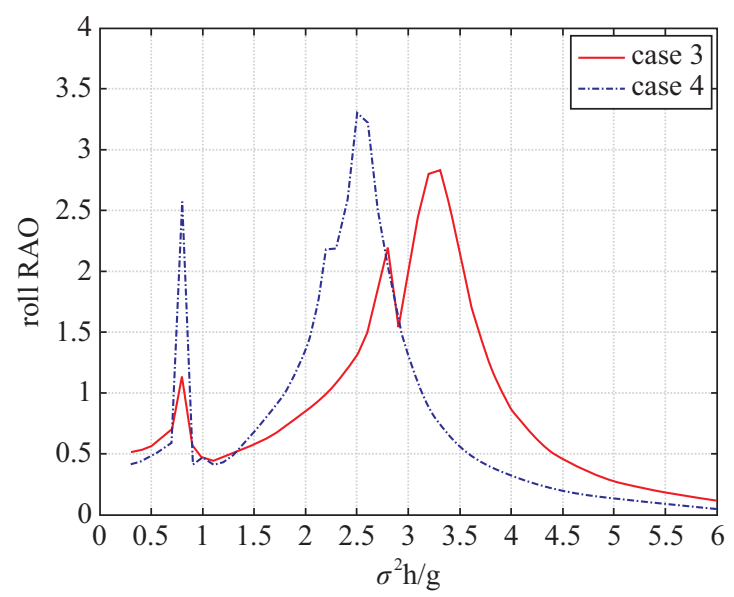

Fig. 8. Comparison of roll RAO with different drafts.

is considered as linear and symmetric, and the hydrodynamic forces on the mooring line are neglected. This model is developed by BIEM, and the free surface nodes are tracked by MEL with cubic spline scheme and RK4. Two damping zones 


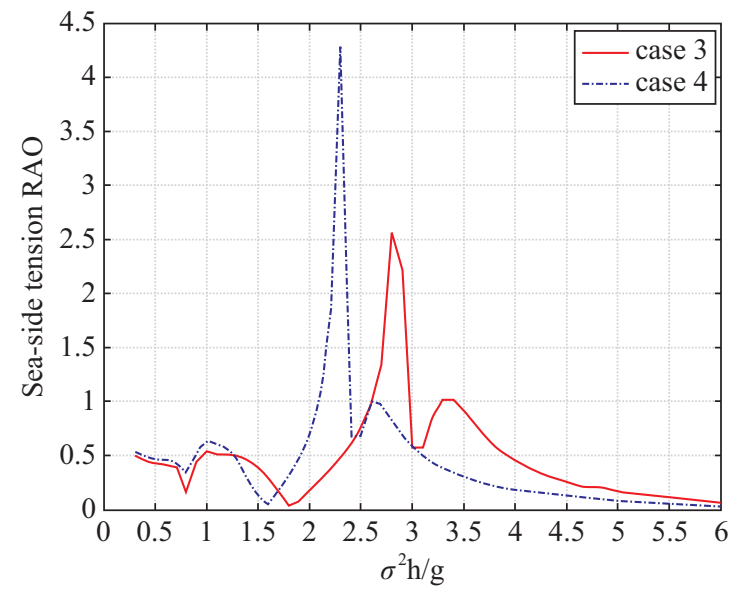

Fig. 9. Comparison of Sea-side tension RAO with different drafts.

are arranged on both ends of NWT to absorb reflected wave energy scattered from the body and to dissipate the transmitted wave energy passing through the body. The simulation of floating body motion is calculated by the acceleration potential method and the mode-decomposition method. The results indicate that the liquid container has influences on the structure dynamic responses. The resonant frequencies of DPFS with liquid water slightly downshift but with higher peak values when comparing to DPFS only. These may be due to the increase of total mass generating downshift phenomena. As for the sea-side tension, the occurrence of the resonant frequency not only downshifts but also reduces significantly, which may be attributed to the mass of liquid container added into the system and this liquid may generate a sloshing effect against the structural movement to mitigate the mooring tension. Finally, the influences of the depth of draft were investigated. The results demonstrate that the deeper draft down shifts the resonant frequencies but generates the higher peak values for dynamic responses. These phenomena can be perceived through the total mass increases as the draft increases and more fluid contact force acting on the structure. This study may set a good foundation for further developing renewable energy devices or damping equipment.

\section{ACKNOWLEDGMENTS}

The authors acknowledge the support in part by AsiaPacific Ocean Research Center, NSYSU, and the National Science Council (NSC98-2221-E-110-087), Taiwan.

\section{REFERENCES}

1. Chakrabarti, S. K., "Wave forces on multiple vertical cylinders," Journal of the Waterway Port, Coastal and Ocean Division, Vol. 104, No. 2, pp. 147-161 (1978).

2. Chen, J. T., Lee, Y. T., and Lin, Y. J., "Interaction of water waves with arbitrary vertical cylinders using null-field integral equations," Applied Ocean Research, Vol. 31, pp. 101-110 (2009).

3. Cointe, R., "Numerical simulation of a wave channel," Engineering Analysis with Boundary Elements, Vol. 7, No. 4, pp. 167-177 (1990).

4. Gao, H., Kwok, K. C. S., and Samali, B., "Optimization of tuned liquid column dampers," Engineering Structures, Vol. 19, No. 6, pp. 476-486 (1997).

5. Grilli, S. T. and Svendsen, I. A., "Corner problems and global accuracy in the boundary element solution of nonlinear wave flows," Engineering Analysis with Boundary Elements, Vol. 7, No. 4, pp. 178-195 (1990).

6. Hitchcock, P. A., Kwok, K. C. S., and Watkins, R. D., "Characteristics of liquid column vibration absorbers (LCVA)-I," Engineering Structures, Vol. 19, No. 2, pp. 126-134 (1997a).

7. Hitchcock, P. A., Kwok, K. C. S., and Watkins, R. D., "Characteristics of liquid column vibration absorbers (LCVA)-II," Engineering Structures, Vol. 19, No. 2, pp. 135-144 (1997b).

8. Huang, C. C., Tang, H. J., and Chen, W. M., "On the interaction between random waves and a freely floating body in a fully nonlinear numerical wave tank," Proceeding of the 18th International Offshore and Polar Engineering Conference, Vol. 3, pp. 148-155 (2008).

9. Isaacson, M. De St Q., "Nonlinear-wave effects on fixed and floating bodies," Journal of Fluid Mechanics, Vol. 120, pp. 267-281 (1982).

10. Koo, W. C. and Kim, M. H., "Freely floating-body simulation by a 2D fully nonlinear numerical wave tank," Ocean Engineering, Vol. 31, pp. 2011-2046 (2004).

11. Lee, H. H., Wong, S. H., and Lee, R. S., "Response mitigation on the offshore floating platform system with tuned liquid column damper," Ocean Engineering, Vol. 33, pp. 1118-1142 (2005).

12. Longuet-Higgins, M. S. and Cokelet, E., "The deformation of steep surface waves on water: I. a numerical method of computation," Proceedings of Royal Society of London, Series A, Vol. 350, pp. 1-26 (1976).

13. Mac Camy, R. C. and Fuchs, R. A., "Wave force on piles: a diffraction theory," Technical memorandum No. 69, US Army Corps of Engineers, Beach Erosion Board (1954).

14. Nojiri, N. and Murayama, K., "A study on the drift force on twodimensional floating body in regular waves," Transactions of the WestJapan Society Naval Architect, Vol. 51, pp. 131-152 (1975).

15. Perrey-Debain, E., Trevelyan, J., and Bettess, P., "Plane wave interpolation in direct collocation boundary element method for radiation and wave scattering: Numerical aspects and applications," Journal of Sound and Vibration, Vol. 261, pp. 839-858 (2003).

16. Tang, H. J. and Huang, C. C., "Bragg reflection in a fully nonlinear numerical wave tank based on boundary integral equation method," Ocean Engineering, Vol. 35, pp. 1800-1810 (2008).

17. Tanizawa, K., "A nonlinear simulation method of 3-D body motions in waves (1st Report)," Journal of the Society of Naval Architect Japan, Vol. 178, pp. 179-191 (1995).

18. Tanizawa, K., "Long time fully nonlinear simulation of floating body motions with artificial damping zone," Journal of the Society of Naval Architects of Japan, Vol. 180, pp. 311-319 (1996).

19. Tanizawa, K. and Minami, M., "On the accuracy of NWT for radiation and diffraction problem," Abstract for the 6th Symposium on Nonlinear and Free-surface Flow (1998).

20. Vinje, T. and Brevig, P., "Nonlinear ship motions," Proceedings of the 3rd International Conference on Numerical Ship Hydrodynamics, IV3, pp. 1-10 (1981).

21. Weng, W. K. and Chou, C. R., "Analysis of response of floating dual pontoon structure," China Ocean Engineering, Vol. 21, No. 1, pp. 91-104 (2007).

22. Williams, A. N. and Abul-Azm, A. G., "Dual pontoon floating breakwater," Ocean Engineering, Vol. 24, No. 5, pp. 465-478 (1997). 